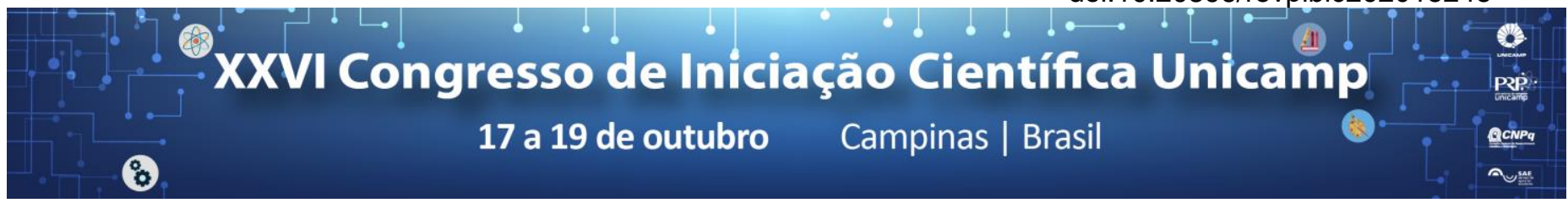

\title{
Oscillations and chaos in electrochemical interfaces
}

\section{Caio da Silva Rodrigues, Renan C. C. de Miranda, Raphael Nagao*}

\begin{abstract}
A prototype of an electrochemical oscillator is represented by three nonlinear differential equations. Through numerical computation it is possible to study the system by calculating the time series and the respective Lyapunov exponents which describes whether the system is periodic or chaotic.
\end{abstract}

\section{Key words:}

time series, electrochemical oscillations, chaos.

\section{Introduction}

It is possible to observe oscillatory behavior in the most diverse situations of the human routine, for example, in organic systems as the biological rhythm and in non-living ones, like those of economic origin. ${ }^{[1]}$ The same occurs in electrochemical systems, where periodic and chaotic behavior can be wildly observed in determined values of the controlling parameters. ${ }^{[2]}$ The electrochemical interface was modeled by three ordinary differential equations (ODE) that describe the time evolution of the double layer potential $(\phi)$, the concentration of electroactive species (c) and surface coverage of an inhibiting adsorbate $(\theta)^{[1,2]}$. We calculated this set of ODE by implementing a fourth order Runge-Kutta numerical method and calculate the Lyapunov exponent $(\lambda)$, based on an algorithm developed by Wolf et. al. ${ }^{[3]}$, to characterize the nonlinear time series. It basically represents the speed at which two nearby points tend to move away or approach in the phase space. $\lambda$ is positive if the time series is aperiodic and negative for periodic behavior. If the sum of the exponents is zero, the system is considerate as conservative.

\section{Results and Discussion}

Figure 1a represents mixed mode oscillations for the controlling parameters: resistance $(\rho)$ of 106.97 and applied voltage $(U)$ of 246.15 . Note that our model is dimensionless. For these parameter values, the time series shown in plate (a) has a periodicity, that is, with each time interval $(\Delta t)$, it is realized that the peaks of the undulations are repeated. The attractor (Figure 1b), calculated by plotting two independent variables, namely $\theta$ vs. $\phi$, has four turns that represents the period $1^{3}$ (notation $L^{S}$ for large $(L$ $=1$ ) and small $(S=3)$ amplitudes) for the parameters used in the calculation. The smallest Lyapunov exponent $(\lambda)$ showed a value of $\lambda=-0.0455$ bits/s which corroborates with a periodic behavior. Nevertheless, with subtle changes in $U$ and $\rho$ a chaotic time series was obtained, see Figure 1c. The parameters utilized in the calculation are: $\rho=$ 106.75 and $U=246$. The time series did not show repeatability for any time interval. The attractor is called strange (Figure 1d) and it is more difficult to observe how many trajectories it has, showing a fractal geometry. In this case the largest Lyapunov exponent showed a positive value of $\lambda=+0.125$ bits $/ \mathrm{s}$. Although the values agree, we are still working to get the best values for our time series. (a)

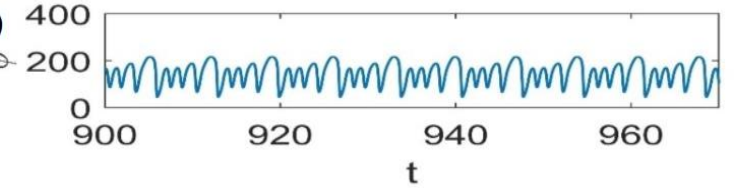

(b)

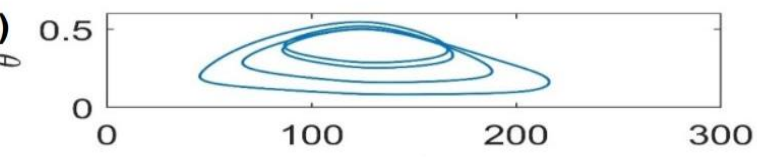

(c)

$\phi$
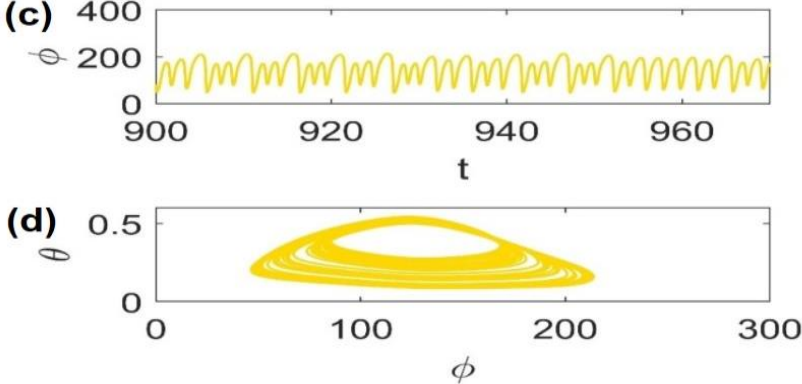

Figure 1. Time series for (a) periodic and (b) chaotic system. (c) (d) They are their respective attractors.

\section{Conclusions}

Through numerical simulations, it was possible to analyze the regions where the system presented chaotic or periodic characteristics. The time series were also analyzed by the reconstruction of the attractors by plotting two independent variables. We calculated the Lyapunov exponent for both behaviors and although the values agree, we are still working to get the results already reported in the literature.

\section{Acknowledgement}

The authors acknowledge SAE and CAPES for the scholarships and FAPESP (\#2016/01817-9) for financial support.

\section{References}

${ }^{1}$ Batista, B. C. Tese de Doutorado, Efeito do envenenamento superficial da dinâmica de osciladores eletroquímicos: Experimento, modelagens e simulações. São Carlos, 2013, p.4.

${ }^{2}$ Nascimento, M. A.; Gallas, J. A. C.; Varela, H. Self-organized distribution of periodicity and chaos in an eletrochemical oscillator. Phys. Chem. Chem. Phys. 2011, p.442. 